\title{
Transverse Collective Flow of Hadrons at RHIC and at LHC
}

\section{P. K. Khandai ${ }^{*}{ }^{1}$, and P. Shukla ${ }^{2}$}

1Department of Physics, Ewing Christian College, Allahabad, 211003, India

1Department of Physics, NIT Jamshedpur, Jharkhand, 831014, India

2Nuclear Physics Division, Bhabha Atomic Research Center, Mumbai 400085, India

E-mail: pkkhandai@gmail.com and pshuklabarc@gmail.com

We present a systematic study of transverse momentum $\left(p_{T}\right)$ spectra of hadrons produced in $p+p$ and in heavy ion collisions at RHIC and LHC energies. The hadron spectra in $p+p$ collisions can be explained by Tsallis distribution with two parameters ( $T$ and $q$ ). To explain the hadron spectra in heavy ion collisions in a wider $p_{T}$ range we use a modified Tsallis function by introducing an additional parameter $(\beta)$ which accounts for collective transverse flow. The new analytical function gives a very good description of both mesons and baryons spectra at all centralities of $A u+A u$ collisions at $\sqrt{s_{N N}}=200 \mathrm{GeV}$ and $P b+P b$ collisions at $\sqrt{s_{N N}}=$ $2.76 \mathrm{TeV}$. The parameter representing transverse collective flow increases with system size and is found to be more for baryons than that for mesons in more central $A u+A u$ collisions. The non-thermalization parameter $(q)$ decreases with centrality in heavy ion collisions which gives an indication of thermalization in heavy ion collisions at both RHIC and LHC.

7th International Conference on Physics and Astrophysics of Quark Gluon Plasma,

1-5 February, 2015

Kolkata, India

* Speaker. 


\section{Introduction}

The aim of the heavy ion collisions at Relativistic Heavy Ion Collider (RHIC) and Large Hadron Collider (LHC) is to create and study a system of strongly interacting matter at high energy density [1]. The measurements in $p+p$ collisions are important to understand the particle production mechanism [2] and are also used as baseline for heavy ion (HI) collisions. Hadrons (pions, kaons and protons) form the bulk of particles measured in a heavy ion collision experiment. Transverse momentum $\left(p_{T}\right)$ distributions of identified hadrons are the most common tools used to study the dynamics of high energy collisions. The hadron spectra at high transverse momentum $\left(p_{T}\right)$ arise from the jets from initial hard partonic collisions and are known to follow a power law distribution. Jets are suppressed [3] in the Quark Gluon Plasma (QGP), an effect menifested in high $p_{T}$ part of hadron spectra. The low $p_{T}$ hadrons form the bulk of the spectra arising from multiple scatterings follow exponential distribution which for heavy ion systems is depictive of particle distribution in a thermal system. In addition, for heavy ion systems the hadron spectra at intermediate $p_{T}$ is affected by transeverse flow and/or quark recombination $[4,5]$.

The Tsallis distribution [6,7] describes near thermal systems in terms of two parameters; temperature and $q$ which measures temperature fluctuation or degree of non-thermalization. In a recent work [8], we used the Tsallis distribution to describe the $p_{T}$ spectra of identified charged hadrons measured in $p+p$ collisions at RHIC $(\sqrt{s}=62.4,200 \mathrm{GeV})$ and at $\mathrm{LHC}(\sqrt{s}=0.9,2.76$ and $7.0 \mathrm{TeV}$ ) energies. It has been shown $[7,8]$ that the functional form of the Tsallis distribution with thermodynamic origin is the same as the form of QCD inspired Hagedorn distribution $[9,10]$. The parameter $q$ of Tsallis is thus related to the power $n$ of the Hagedorn function characterizing hard hadronic collisions.

The shape of the $p_{T}$ distributions of mesons and baryons resemble the $p_{T}$ distribution of quarks as shown by recombination models [11]. Thus, the change in the $p_{T}$ spectra of quarks due to collective flow etc. will be reflected in the measured $p_{T}$ distribution of hadrons in heavy ion collisions which could be included in phenomenological fit functions such as the Hagedorn or Tsallis formula. To explain the hadron spectra in heavy ion collisions in a larger $p_{T}$ range, a new modified Tsallis function [12] was proposed by introducing an additional parameter which accounts for transverse flow. In this work, we make a systematic study of parameters of modified Tsallis distribution for different hadrons produced in RHIC $A u+A u$ collisions at $\sqrt{s_{N N}}=200 \mathrm{GeV}$ and in LHC $P b+P b$ collisions at $\sqrt{s_{N N}}=2.76 \mathrm{TeV}$.

\section{The modified Tsallis distribution}

The Tsallis distribution $[6,7]$ describes a near thermal system in terms of two parameters $T$ and $q$ and is given by

$$
E \frac{d^{3} N}{d p^{3}}=C_{n}\left(1+\frac{E}{n T}\right)^{-n}
$$

Here $E$ is the particle energy, $T$ is the temperature and $n=1 /(q-1)$ with $q$ being the nonextensivity parameter which measures the temperature fluctuations [13] in the system as: $q-1=\operatorname{Var}(T) /<$ 
$T>^{2}$. The values of $q$ lie between $1<q<4 / 3$. Larger values of $q$ correspond to smaller values of $n$ describing a system away from thermal equilibrium. In terms of QCD, smaller values of $n$ imply dominant hard point-like scattering. Phenomenological studies suggest that, for quark-quark point scattering, $n \sim 4[14,15]$, and when multiple scattering centers are involved $n$ grows larger and can become large.

There have been many attempts to use Tsallis distributions in hydrodynamical models [e.g. [16]]. These formalisms could be used to explain the hadronic spectra produced in heavy ion collisions in the low $p_{T}$ range. We use an analytical fit function which works in a wider $p_{T}$ range and is given by

$$
E \frac{d^{3} N}{d p^{3}}=C_{n}\left(\exp \left(\frac{-\gamma \beta p_{T}}{n T}\right)+\frac{\gamma m_{T}}{n T}\right)^{-n}
$$

The low and high $p_{T}$ limits of this formula are given by

$$
\begin{aligned}
E \frac{d^{3} N}{d p^{3}} \simeq\left(1+\frac{\gamma\left(m_{T}-\beta p_{T}\right)}{n T}\right)^{-n} & \\
E \frac{d^{3} N}{d p^{3}} & \simeq\left(1+\frac{\gamma\left(m_{T}-\beta p_{T}\right)}{n T}\right)^{-n} \\
& \simeq \exp \left(\frac{-\gamma\left(m_{T}-\beta p_{T}\right)}{T}\right) \text { for } p_{T} \rightarrow 0 \\
& \simeq\left(\frac{\gamma m_{T}}{n T}\right)^{-n} \text { for } p_{T} \rightarrow \infty .
\end{aligned}
$$

At low $p_{T}$, it represents a thermalized system with collective flow and at high $p_{T}$ it becomes a power law which mimics "QCD inspired" quark interchange model [10].

We apply the formula (Eq. 2.2) on transverse momentum spectra measured in $p+p$ and $A u+$ $A u$ collisions at $\sqrt{s_{N N}}=200 \mathrm{GeV}$ and also in $P b+P b$ collisions at $\sqrt{s_{N N}}=2.76 \mathrm{TeV}$. Here we use all the data from PHENIX $(|y|<0.35)[17,18,19,20]$, STAR $(|y|<0.5,|y|<0.75)[21,22,23,24]$ and ALICE $(|y|<0.5)[25]$ experiments. The errors on the data are quadratic sums of statistical and uncorrelated systematic errors. The particle spectra are studied as a function of system size using $p+p, A u+A u$ and $P b+P b$ collisions for different centralities.

\section{Results and Discussions}

In this analysis, first we fit all the measured spectra of pions, kaons, protons, $\Lambda$ and $\Xi$ in $p+p$ collisions at $\sqrt{s_{N N}}=200 \mathrm{GeV}$ using modified Tsallis distribution (Eq. 2.2) with same temperature to obtain different values of $n$ for different particles. The value of $\beta$ are small in $p+p$ collisions as is expected. The spectra of different particles in $A u+A u$ collisions for all centralities are fitted to obtain $\beta$ and $T$ as a function of system size. Figure 1 shows the variation of the transverse flow velocity $\beta$ and the freeze-out temperature $T$ for pions, kaons, protons, $\Lambda$ and $\Xi$ for $p+p$ and different centralities of $A u+A u$ collisions at $\sqrt{s_{N N}}=200 \mathrm{GeV}$. We observe that the flow velocity $(\beta)$ for all particles is very small in $p+p$ collisions and increases with centrality in $A u+A u$ collisions which is expected. For central collisions, there is a clear separation of flow velocity between mesons and baryons. For the most central $A u+A u$ collisions, the values of $\beta$ for protons is around 

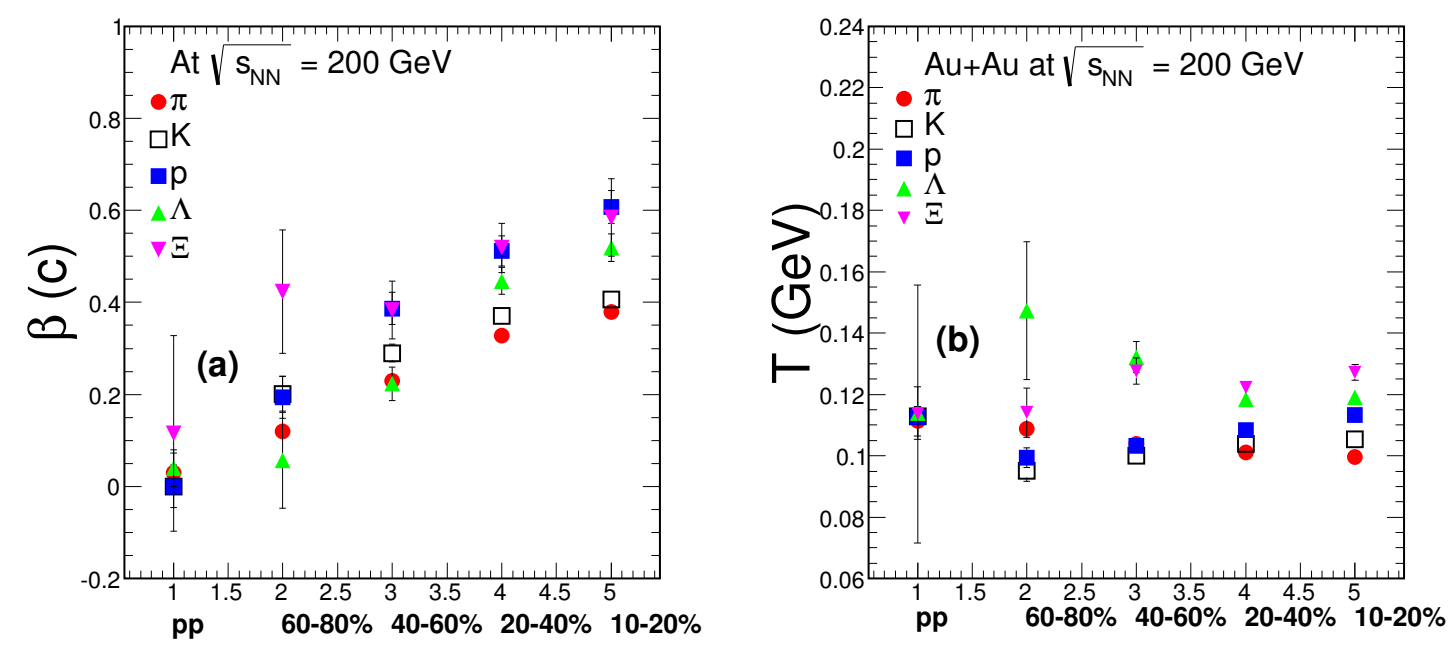

Figure 1: The left panel shows the variation of transverse flow velocity $\beta$ and the right panel shows the variation of freeze-out temperature $T$ as a function of collision system size at $\sqrt{s_{N N}}=200 \mathrm{GeV}$ for pions $[17,18,19,20]$, kaons [17, 19], protons [21, 23], $\Lambda[22,24]$ and $\Xi[22,24]$.

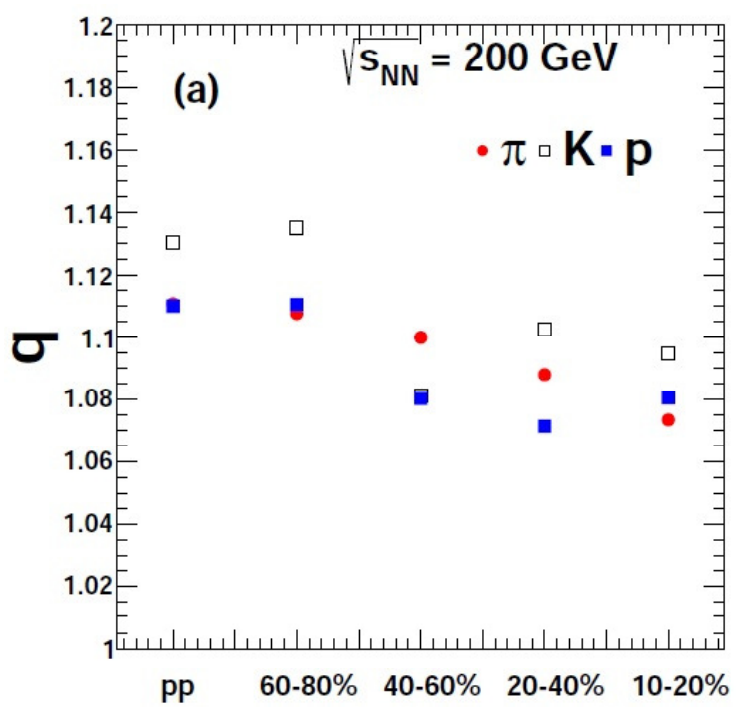

Figure 2: The variation of parameter $q$ as a function of system size at $\sqrt{s_{N N}}=200 \mathrm{GeV}$ for pions, kaons and protons. 


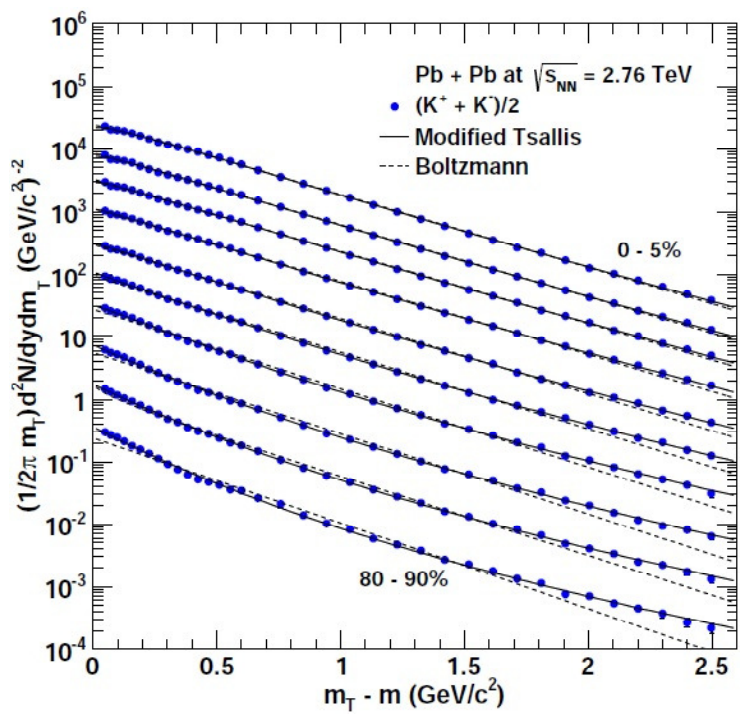

Figure 3: The invariant yields of kaons $\left(\left(K^{+}+K^{-}\right) / 2\right)$ [25] as a function of $m_{T}$ for $P b+P b$ collisions at $\sqrt{s}$ $=2.76 \mathrm{TeV}$ for various centralities (from top to bottom $0-5 \%, 5-10 \%, 10-20 \%, 20-30 \%, 30-40 \%, 40-50 \%$, $50-60 \%, 60-70 \%, 70-80 \%, 80-90 \%$ ). The solid lines are the modified Tsallis distribution (Eq. 2.2) and the dotted lines are the Boltzmann distribution.
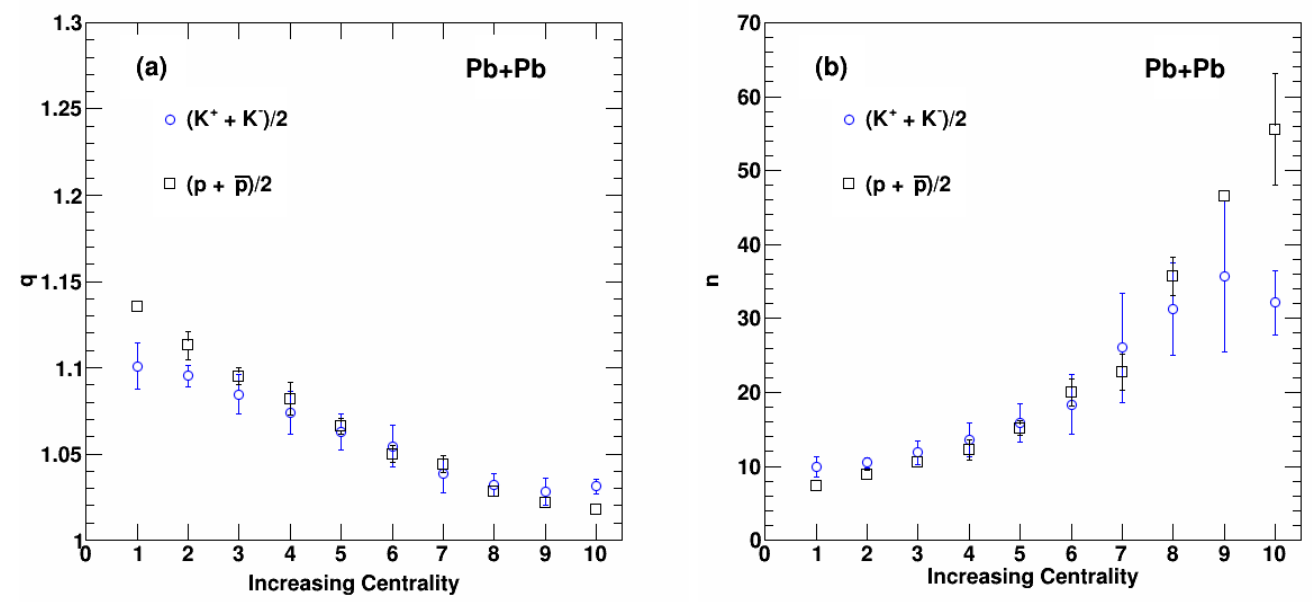

Figure 4: The left panel (a) shows the variation of parameter $q$ and the right panel (b) shows the variation of parameter $n$ in $P b+P b$ collisions at $\sqrt{s_{N N}}=2.76 \mathrm{TeV}$ of kaons [25] and protons [25] for various centralities. Here the values of centralities are shown as $1,2,3 \ldots \ldots .10$ should read as $0-5 \%, 5-10 \%, 10-20 \%, \ldots \ldots . .80-90 \%$. 
0.6 and for pions it is 0.4 , which shows a dependence on number of constituent quarks. The baryons in general freeze out earlier than the mesons for central collisions.

Figure 2 shows the variation of parameter $q$ as a function of system size at $\sqrt{s_{N N}}=200 \mathrm{GeV}$ for pions, kaons and protons. Here we see that the non-thermalization parameter $(q)$ decreases with centrality implying a more thermalized system for central collisions.

Figure 3 shows the plot of invariant yields of kaons $\left(\left(K^{+}+K^{-}\right) / 2\right)$ as a function of $m_{T}$ for $P b+P b$ collisions at $\sqrt{s}=2.76 \mathrm{TeV}$ for various centralities. The solid lines are the modified Tsallis distributions (Eq. 2.2) and the dotted lines are the Boltzmann distributions. Here it is clearly seen that the modified Tsallis distribution approaches Boltzmann distribution with increasing system size, representing a typical thermal system.

Figure 4 (a) and (b) respectively show the variations of parameters $q$ and $n$ in $P b+P b$ collisions at $\sqrt{s_{N N}}=2.76 \mathrm{TeV}$ for kaons and protons at various centralities. The decreasing value of $q$ or increasing value of $n$ imply that the system is increasingly thermalized for more central collisions.

\section{Conclusions}

This work presents a systematic study of transverse momentum $\left(p_{T}\right)$ spectra of hadrons produced in $p+p$ and in heavy ion collisions at both RHIC and LHC energies. To describe the hadron spectra in heavy ion collisions in a wider $p_{T}$ range we use a modified Tsallis function with an additional parameter $(\beta)$ which accounts for collective transverse flow. The analytical function gives a very good description of both mesons and baryons spectra at all centralities of $A u+A u$ collisions at $\sqrt{s_{N N}}=200 \mathrm{GeV}$ and $P b+P b$ collisions at $\sqrt{s_{N N}}=2.76 \mathrm{TeV}$. The parameter representing transverse collective flow increases with system size and is found to be more for baryons than that for mesons in central $A u+A u$ collisions. The non-thermalization parameter $(q)$ decreases with centrality in heavy ion collisions which gives an indication of thermalization in heavy ion collisions at both RHIC and LHC.

\section{References}

[1] K. Adcox et. al. (PHENIX), Nucl. Phys. A757, 184 (2005); J. Adams et al. (STAR), Nucl. Phys. A757, 102 (2005); N. Armesto et al., J. Phys. G35, 054001 (2008).

[2] F. Becattini and U. Heinz, Z. Phys. C76, 269 (1997).

[3] X. Wang, Phys.Lett. B 579, 299 (2004).

[4] R. J. Fries, V. Greco, P. Sorensen, Ann. Rev. Nucl. Part. Sci. 58, 177 (2008).

[5] R. Fries, J. Phys. G30, S853 (2004).

[6] C. Tsallis J. Stat. Phys. 52479 (1988).

[7] T. S. Biro, G. Purcsel, K. Urmossy Eur.Phys.J. A40 325 (2009).

[8] P. K. Khandai , P. Sett, P. Shukla and V. Singh Int. J. Mod. Phys. 28, 1350066 (2013).

[9] R. Hagedorn Rev. del Nuovo Cim. 6N 101 (1984).

[10] R. Blankenbecler and S. J. Brodsky Phys. Rev. D 102973 (1974).

[11] V. Greco, C. M. Ko and P. Levai Phys. Rev. C 68034904 (2003). 
[12] P. K. Khandai, P. sett, P. Shukla and V. Singh J. Phys. G: Nucl. Part. Phys. 41025105 (2014).

[13] G. Wilk and Z. Wlodarczyk Phys. Rev. Lett. 842770 (2000).

[14] R. Blankenbecler, S. J. Brodsky and J. Gunion Phys. Rev. D 123469 (1975).

[15] S. J. Brodsky, H. J. Pirner and J. Raufeisen Phys. Lett. B 63758 (2006).

[16] M. Shao,L. Y. Zebo Tang, H. Chen,C. Li and Z. Xu J. Phys. G 3785104 (2010).

[17] S. S. Adare et al PHENIX Collaboration Phys. Rev. C 74024904 (2006).

[18] A. Adare et al PHENIX Collaboration Phys. Rev. D 76051106 (2007).

[19] S. S. Adler et al PHENIX Collaboration Phys. Rev. C 69034909 (2004).

[20] A. Adare et al PHENIX Collaboration Phys. Rev. Lett. 101232301 (2008).

[21] B. I. Abelev et al STAR Collaboration Phys. Rev. C 7564901 (2007).

[22] J. Adams et al STAR Collaboration Phys. Lett. B 637161 (2006).

[23] J. Adams et al STAR Collaboration Phys. Rev. Lett. 97132301 (2006).

[24] J. Adams et al STAR Collaboration Phys. Rev. Lett. 9862301 (2007).

[25] B. Abelev et al (ALICE Collaboration) Phys. Rev. C 88, 044910 (2013). 